\title{
Morphological observations on patients with presumed trichiasis
}

\author{
K BARBER AND T DABBS \\ From the Department of Ophthalmology, Royal Hallamshire Hospital, Sheffield S10 $2 \mathrm{JF}$
}

SUMmaRY The clinical findings in 116 patients with a provisional diagnosis of trichiasis are presented. In $69 \%$ a small degree of entropion producing lash-globe contact was detected. The term lid border entropion is proposed for this condition, which is recognised clinically by conjunctivalisation of the meibomian gland orifices and anterior placement of the mucocutaneous junction. These features were clearly demonstrated by electron microscopy of a biopsy specimen from one patient. An analysis of other causes of trichiasis is discussed and an attempt is made to clarify the terminology currently in use.

A number of early descriptions of trichiasis exist, mainly owing to its association with trachoma.' The Ebers Papyrus which dates back to the 16th century $\mathrm{BC}$ describes it, as did Hippocrates in the 5th century BC. Despite this historical background, trichiasis has no universally accepted specific definition and can be used for a number of eyelid or eyelash abnormalities. Many terms are used to describe it, including: supernumerary lashes, metaplastic lashes, aberrant lashes, pseudocilia, and distichiasis. It is probably best regarded as an 'umbrella term' for conditions characterised by lash-globe contact.

A need exists to describe the morphology of trichiasis and to remove some of the confusion on its terminology. We present here a study of the clinical characteristics and morphology of a group of patients with presumed trichiasis.

\section{Patients and methods}

One hundred and sixteen patients regularly attending an ophthalmic unit with a provisional diagnosis of trichiasis were entered into the study. They were all undergoing intermittent epilation by nursing staff or specific treatment by medical staff.

Most of the patients attended on a casual basis without appointment and, to estimate the demand at the unit for treatment, details of their age, duration of symptoms, and attendance rates were compiled. Each patient was examined and photographed.

Correspondence to K Barber, FRCS Ed, Worcester Eye Hospital, Barbourne Road, Worcester.
Face and eyelid photographs were taken to show anatomical details and to provide an accurate record.

\section{Results}

The age of the patients varied from 20 to 89 years, with an average of 64 years. The onset of trichiasis for each patient was noted and a histogram compiled. The onset of trichiasis was maximal between the ages of 60 and 70 years (Fig. 1). The average duration for trichiasis in this group of patients is shown in Fig. 2: $58 \%$ had suffered from trichiasis for five years or more and $15 \%$ for 30 years or more. The patients were attending an ophthalmic casualty, usually for epilation on a regular basis by nursing staff; $40 \%$

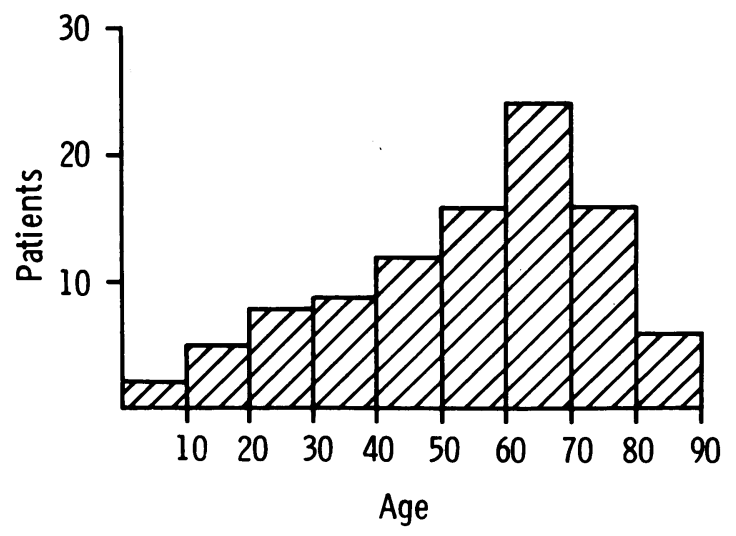

Fig. 1 Age distribution of patients at onset of trichiasis. 


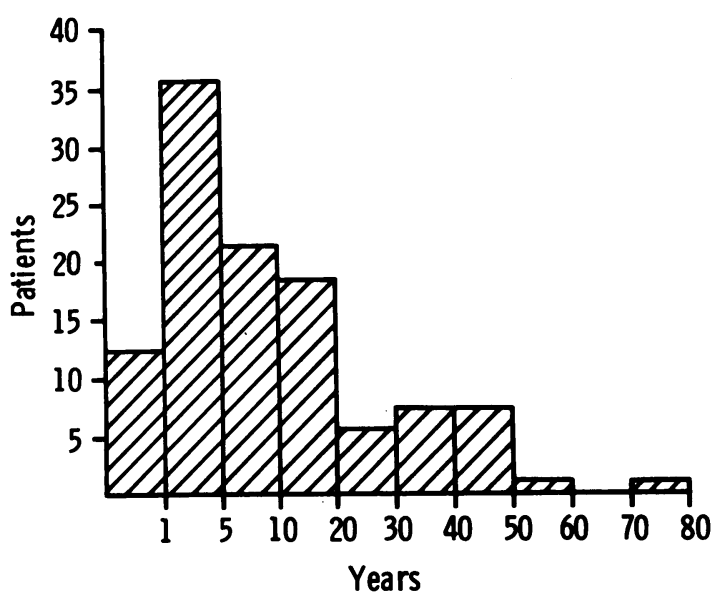

Fig. 2 Duration in years of trichiasis.

were attending between one and four times per month. The average rate of attendance was once every 7.5 weeks, and this resulted in approximately 10 patient visits per week.

Table 1 shows the distribution of morphological changes seen in this group of 116 patients. Seven

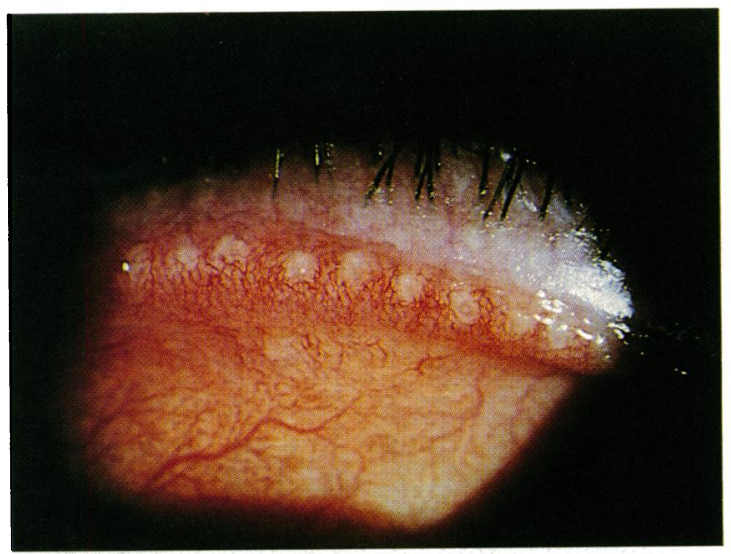

Fig. 3 Upper eyelid with conjunctivalisation of meibomian gland orifices.

patients $(6 \%)$ who regularly attended for epilation by nursing staff complained of irritation and believed that this was due to trichiasis. On successive slit-lamp examinations these patients had no evidence of lashglobe contact despite their symptoms.

The remaining 109 patients could be divided into
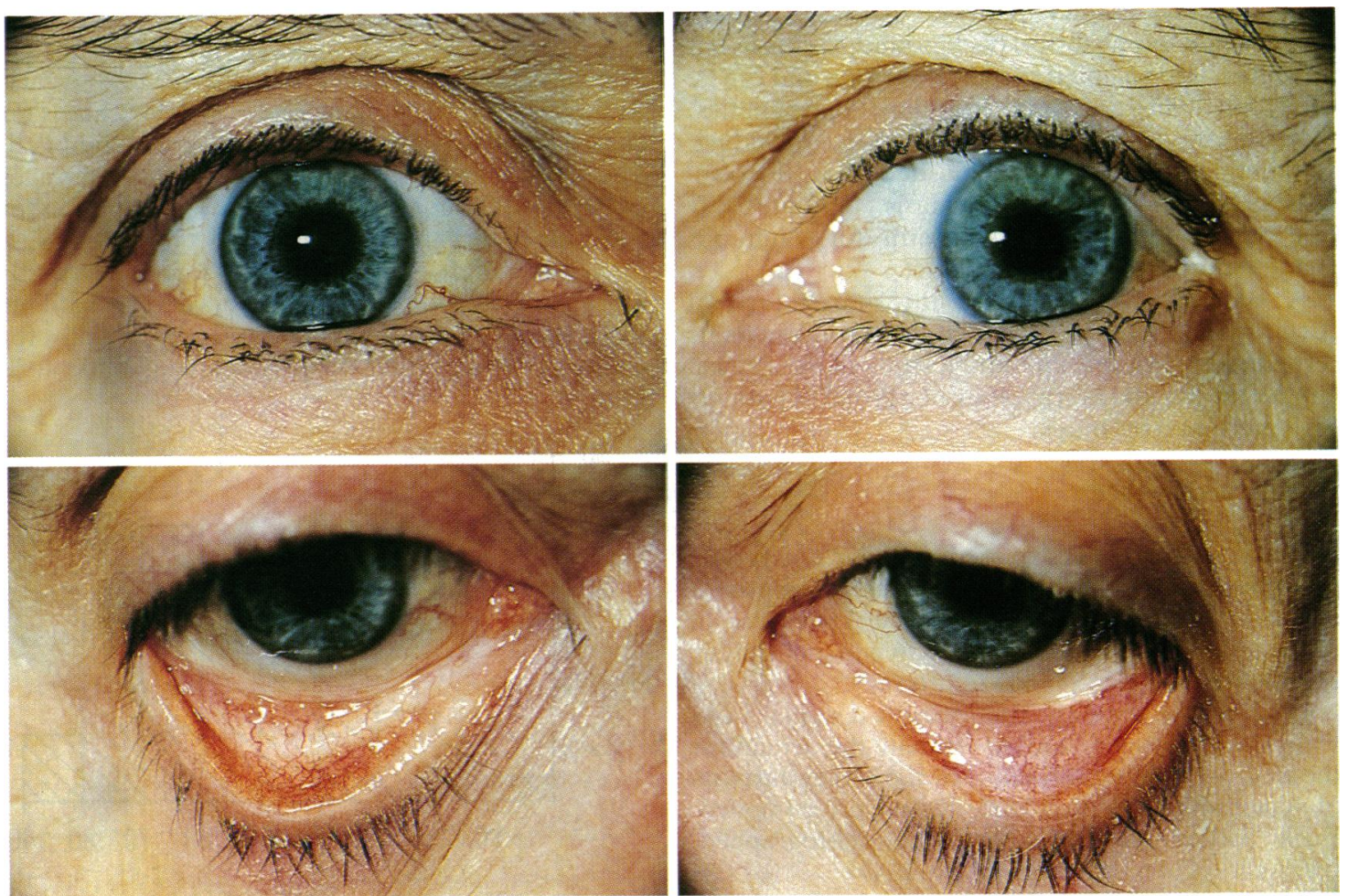

Fig. 4 Left lower lid trichiasis with conjunctival scarring and lid border entropion (compare with normal right eye). 


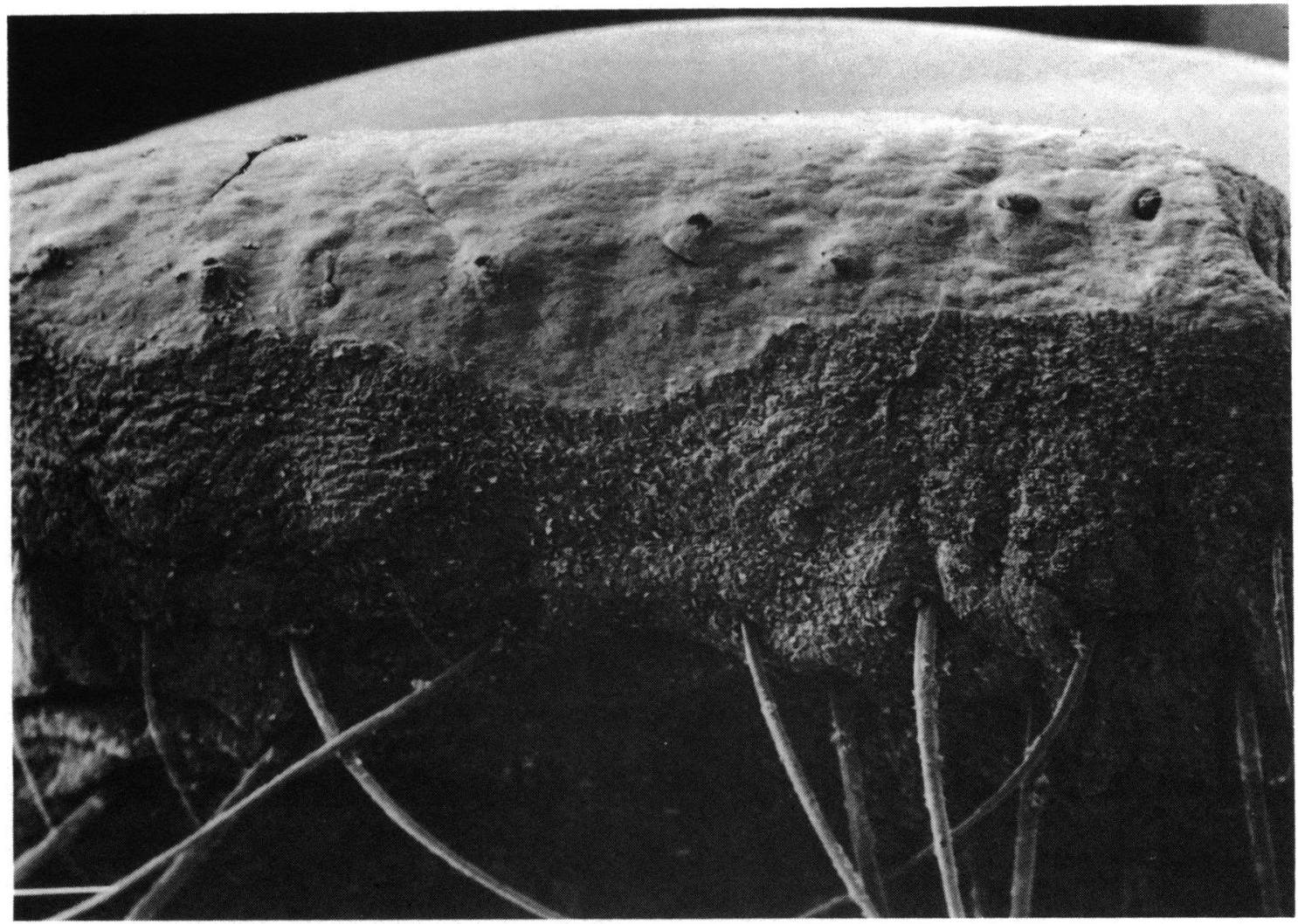

Fig. 5A

Fig. 5A: Scanning electronmicrograph of a block of eyelid from a patient with lid and border entropion. The mucocutaneous junction is seen between the meibomian gland orifices and the lash line. B: Mucocutaneous junction showing division between squamous epithelium and conjunctival epithelium. C: Microvilli on conjunctival epithelium.

those patients with eyelash abnormalities causing lash-globe contact and those with eyelid abnormalities doing so. Only 14 patients had purely eyelash abnormalities. The great majority of patients (95) had eyelid abnormalities causing lash-globe contact. Seven patients had misdirection of lashes that were causing lash-globe contact, with an otherwise normal eyelid margin. Misdirected lashes were seen together with lid border entropion in 29 patients and in association with lid margin notch in 16 patients.

Table 1 Anatomical abnormalities seen in 116 patients

\begin{tabular}{llr}
\hline No abnormality & Melash abnormalities: Misdirected & 7 \\
& Metaplastic & 7 \\
& Distichiasis & 3 \\
& Malposition & 1 \\
Eyelid abnormalities: & Lid border entropion & 3 \\
& Lid border entropion and & 78 \\
& lid notch & 2 \\
& Lid margin notch & 15 \\
\hline
\end{tabular}

Misdirected lashes are therefore commonly seen in trichiasis but are usually associated with other eyelid abnormalities rather than existing on their own.

Distichiasis (defined as a congenital row of lashes arising from the meibomian gland orifices) was seen in only one patient. Metaplastic lashes (defined as an acquired row of lashes arising from the meibomian gland orifices) were seen in three patients. Another three patients had an extension of the normal eyelash line medially and laterally involving the canthal regions. Recognising this as a cause of trichiasis is probably not justified.

In 95 patients (82\%) a lid abnormality was present. Seventeen patients had lid margin notches secondary to temporary tarsorrhaphy or removal of a lid tumour. In these patients a normal lash line was present except in the notched area, where distortion of the follicles resulted in misdirected lashes.

The most common lid abnormality consisted of a small degree of entropion, which we refer to as lid border entropion. This condition was identified by conjunctivalisation of the meibomian gland orifices 


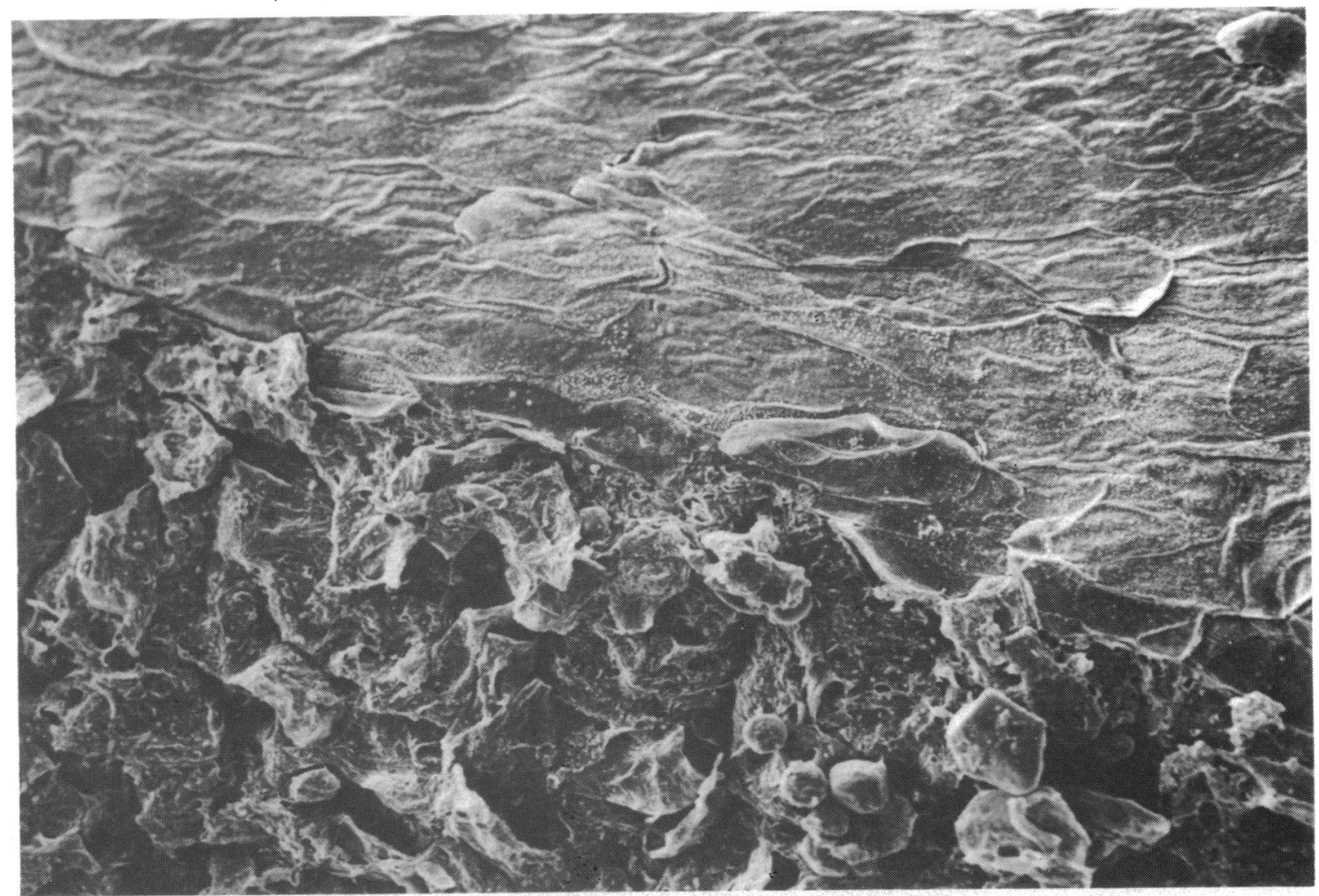

Fig. 5B

and anterior placement of the mucocutaneous border of the lid (Fig. 3).

This is distinguishable from established involutional lower lid entropion in that it is neither intermittent nor invoked by forced closure of the eyelids. Fig. 4 shows lid border entropion (left eye) with lash globe contact. In this case conjunctival scarring has caused the lid border entropion.

Scanning electron microscopy was used to demonstrate the position of the mucocutaneous junction in lid border entropion and to establish the nature of the epithelium at the lid margin in this condition. A corrective pentagon excision was performed on one patient with lid border entropion. Figs. 5A, B, C show the lid margin of this patient. The mucocutaneous junction is well anterior to the meibomian gland orifices; normal conjunctival epithelium with micro-

Table 2 Types of lid border entropion in 80 patients with trichiasis

Cicatricial upper lid border entropion Cicatricial lower lid border entropion Cicatricial upper and lower lid entropion

Involutional lower lid border entropion Involutional upper lid border entropion villi surrounds the meibomian gland orifices, and the mucocutaneous junction is well demarcated with squamous epithelium in juxtaposition with conjunctival epithelium. There is no apparent transition zone.

Eighty patients $(69 \%)$ had lid border entropion which caused lash-globe contact (Table 2). This was seen in association with conjunctival scarring in 41 patients. These patients were thought, therefore, to have a cicatricial lid border entropion. Thirty-nine patients had lid border entropion without any evidence of conjunctival scarring. This has been termed involutional lid border entropion.

\section{Discussion}

A certain amount of confusion exists regarding the terminology pertaining to trichiasis. Trichiasis is derived from the Greek for hair, and is generally used to denote irritation caused by lash-globe contact. Many associated terms are used to describe the various forms of trichiasis, some of which are helpful and others confusing.

Distichiasis has been well described. ${ }^{2-4}$ It is a congenital condition whereby a second row of lashes exists along the posterior border of the lid margin 


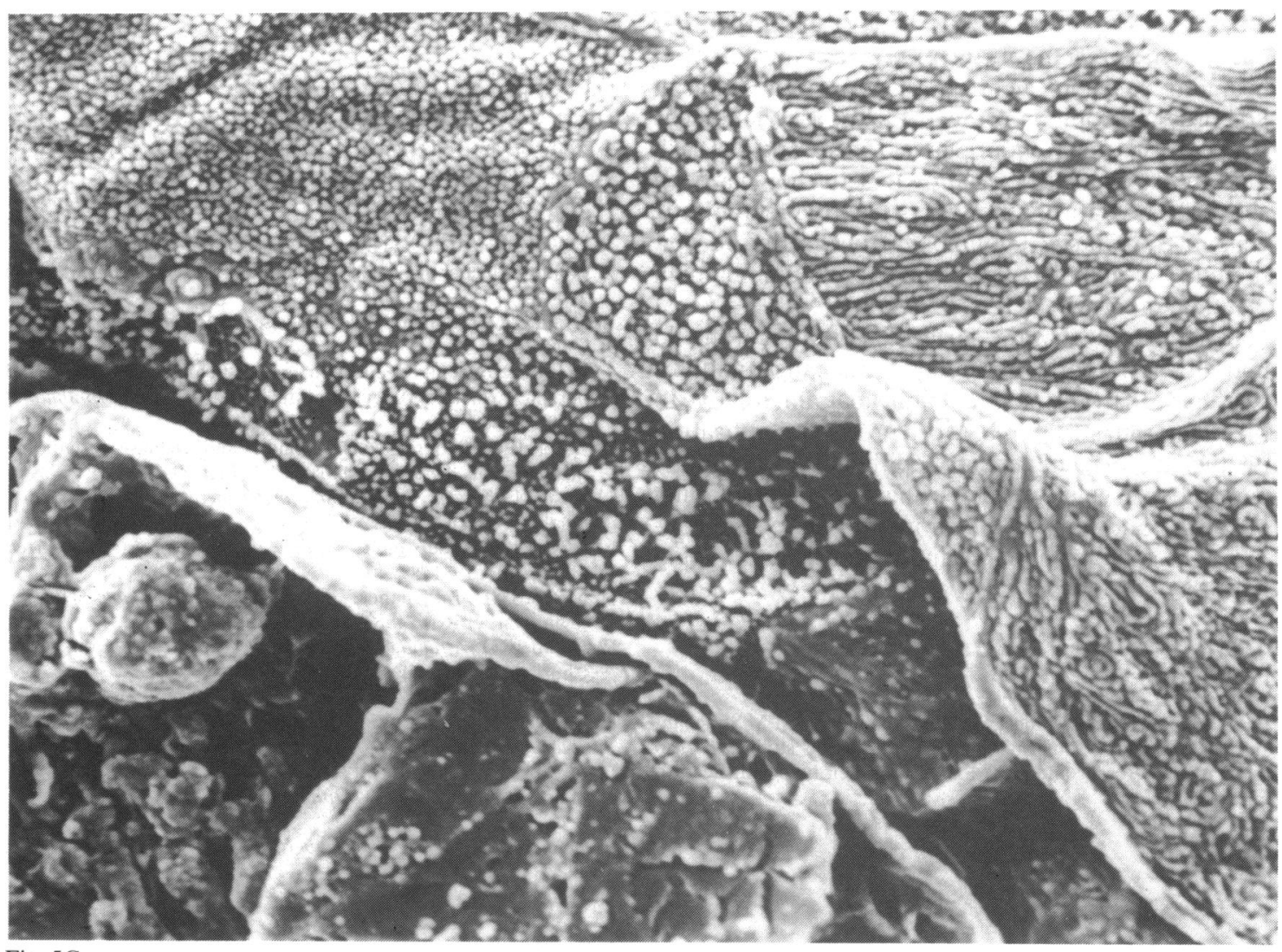

Fig. 5C

in the position of the meibomian gland orifices. Histology of such eyelids has shown lack of meibomian glands. ${ }^{267}$ The meibomian glands are pilosebaceous units in which hair development is absent. ${ }^{+11}$ Failure of differentiation of the pilosebaceous unit results in lashes arising from the meibomian gland region and is regarded as a form of atavism by some. ${ }^{8}$

Under certain circumstances the meibomian glands may revert back to the hair-bearing state of the pilosebaceous unit, and this would then constitute a metaplastic change. It is sensible, therefore, to call these metaplastic lashes. They are seen in association with the late stages of Stevens-Johnson syndrome, in which other metaplastic changes such as keratinisation of the tarsal conjunctiva occur. (Both distichiasis and metaplastic lashes were seen infrequently in this study.)

Misdirected lashes were frequently seen in this study $(45 \%)$. However, they infrequently cause trichiasis by themselves in an otherwise normal eyelid $(6 \%) .14 \%$ of patients had misdirected lashes in combination with lid margin defects. These patients had undergone opening of tarsorrhaphy or tumour removal which involved the lash line without adequate reconstruction of the lid margin.

The majority of patients $(69 \%)$ in this study have a small degree of entropion of the lid border. DukeElder ${ }^{1}$ mentioned this in passing but did not define it, and Duane expressed difficulty in diagnosing entropion in patients with trichiasis." Collin ${ }^{12}$ and Jones et al. ${ }^{13}$ described the early changes of upper lid entropion as an apparent migration of the meibomian gland orifices and conjunctivalisation of the lid margin. This study shows that the entity we refer to as lid border entropion is frequently seen in patients with trichiasis. Clinically it is recognised by the presence of conjunctiva surrounding the meibomian gland orifices, with an abnormal anterior displacement of the mucocutaneous junction. The meibomian gland orifices are easily seen and seem to be prominent when surrounded by conjunctiva. This may be due to the presence of stratified squamous epithelium in the meibomian gland openings, which contrasts with the surrounding conjunctival epithelium.

It is believed that a metaplastic change occurs in surface epithelium, changing keratinised squamous epithelium into conjunctival epithelium with micro- 
villi. The stimulus for this change in lid border entropion is presumably the presence of the tear film. Scanning electron microscopy demonstrates that in lid border entropion the mucocutaneous junction lies between the meibomian gland orifices and the lash line. Interestingly the situation in ectropion has been shown to be entirely the reverse, with a metaplastic change from tarsal conjunctiva to keratinised epithelium taking place posterior to the meibomian gland openings. ${ }^{14}$

It is apparent that the surface epithelium at the lid margin has potential for metaplastic change, and this is influenced by the position of the tear film relative to the lid margin structures. More precise identification of the factors influencing epithelial morphology would be of value in increasing our understanding of those conditions in which metaplasia of the conjunctiva is a prominent and potentially blinding feature.

We thank Miss M A C Jones, Mr C A L Palmer, Mr I M Strachan, and $\mathrm{Mr} \mathrm{J}$ F Talbot for providing patients for this study. We also thank Mr I G Rennie and Dr A Parsons for their help with electron microscopy.

\section{References}

1 Duke-Elder S. Textbook of ophthalmology. St Louis: Mosby, 1952: 5: 383-5.

2 Fox SA. Distichiasis. Am J Ophthalmol 1962; 53: 13-8.

3 Duke-Elder S. Textbook of ophthalmology. St Louis: Mosby, 1952; 3: 873-5.

4 Anderson RL, Harvey JT. Lid splitting and posterior lamellar cryosurgery for congenital and acquired distichiasis. Arch Ophthalmol 1981; 99: 631-4.

5 Kuhnt E. Ueber distichiasis congenita vera. Z Augenheilkd 1899; 2: 46-57.

6 Brailey AR. Congenital distichiasis. Trans Ophthalmol Soc UK 1906; 26: 16-22.

7 Pico S. Congenital ectropion and distichiasis: aetiologic and hereditary factors. A review of the literature. Am J Ophthalmol 1959; 47: 363-87.

8 Scheie HG, Albert DM. Distichiasis and trichiasis: origin and management. Am J Ophthalmol 1966; 61: 718-20.

9 Last RJ. Eugene Wolff's anatomy of the eye and orbit. Philadelphia: Saunders, 1961: 171-2.

10 Wood JR, Anderson R. Complications of cryosurgery. Arch Ophthalmol 1981; 99: 460-3.

11 McCord CD. In: Duane T, ed. Clinical ophthalmology. Hagerstown: Harper Row, 1983: 5: chapter 5: 16-7.

12 Collin JRO. A manual of systematic eyelid surgery. Edinburgh: Churchill Livingstone, 1983: 16.

13 Jones BR, Baulas TC, Hunter PA, Darougar S, Mohsenine H. Neglected lid deformities causing progressive corneal disease. Trans Ophthalmol Soc UK 1976; 96: 45-51.

14 Stefanyszyn MA, Hidayat AA, Flanagan JC. The histopathology of involutional ectropion. Ophthalmology 1985; 92: 120-7.

Accepted for publication 4 December 1986. 